\title{
PARTITIONED BINARY RELATIONS
}

\author{
PAUL MARTIN and VOLODYMYR MAZORCHUK
}

\begin{abstract}
We define the category of partitioned binary relations and show that it contains many classical diagram categories, including categories of binary relations, maps, injective maps, partitions, (oriented) Brauer diagrams and (oriented) Temperley-Lieb diagrams. We construct a one-parameter deformation of the category of partitioned binary relations and show that it gives rise to classical one-parameter deformations of partition, Brauer and Temperley-Lieb categories. Finally, we describe a factorization of partitioned binary relations into a product of certain idempotents and pairs of usual binary relations.
\end{abstract}

\section{Introduction and description of the results}

Diagram algebras and categories are interesting and rich objects of study in modern representation theory with many application to, among others, statistical mechanics, see the book [17] and the surveys [18], [13], and topology, see [24]. Classical diagram categories include the Brauer category (see [3]), the partition category (see [16]), the Temperley-Lieb category (which has many important applications in topology, combinatorics and categorification, see e.g. [26], [2]) and their partial (alias rook) analogues (see [20], [10], [11]), together with the category of binary relations (confer e.g. [23]). From the algebraic perspective all these categories have rich and non-trivial structure, though much less is known for the category of binary relations than the others. Morphisms in these categories are described in terms of certain combinatorially defined sets with diagrammatic realization. Furthermore, most of the classical diagram categories admit a non-trivial one-parameter deformation, which also plays a very important role in certain applications (see e.g. [3]).

The aim of the present paper is to show that both the partition category and the category of binary relations are shadows of a more general natural construction. We define a new category which we call category of partitioned binary relations and show that it provides a single overarching setting for all the categories mentioned above. Our main results are:

- The well-definedness of the new category (Theorem 2.3).

Received 12 October 2011. 
- Connection of the new category with the above mentioned classical objects (Section 3).

- Functorial comparison of the representation theories of the new category and the category of binary relations (Subsection 3.1).

- Well-definedness of a certain flat deformation (Theorem 4.3), which has application in representation theory (confer [4], [5]).

- Factorization of morphisms in the new category in terms of simpler structures (Theorem 5.5).

As a consequence, the partition category and the category of binary relations can be studied simultaneously withing this new bigger object.

A notable feature of our construction is that it is not straightforward. An obvious approach to such an overarching construction is to relax the reflexivesymmetric-transitive condition on the relations that constitute morphisms in the partition category. In fact, this does yield the morphisms in the new category, but it does not determine a composition. Another indication comes from the Temperley-Lieb category, or rather its (topologically motivated) "oriented" generalization (see e.g. [27]). This is easy to extend to the level of the Brauer category and the corresponding partial analogues. The diagrams of this oriented version can be viewed as oriented graphs, which suggests a connection to the category of binary relations. It is worth pointing out that both the partition category and the category of binary relations have also recently appeared in a different context in [6].

The category of binary relations, or rather its endomorphism monoids, are classical objects of study in semigroup theory, see [23], [25], [14] and references therein. In [22] it is shown that every finite group appears as a maximal subgroup of some monoid of binary relations, which shows that these monoids are structurally more complicated than the classical transformation semigroups generalizing the symmetric group (see [9] for the latter).

The paper is organized as follows: In Section 2 we define the category $\mathfrak{B} \mathfrak{B}$ of partitioned binary relations; in Section 3 we show that it contains many classical categories mentioned above; in Section 4 we show that the category $\mathfrak{B} B$ has a flat one-parameter deformation. In Section 5 we describe a factorization of partitioned binary relations, which we call polarized factorization. It turns out that every partitioned binary relation can be written as a product of three elements, two of which are idempotents of a certain simple form, and the third one is a "pair" of usual binary relations. As an application, we show that almost all products of partitioned binary relations result in the full partitioned binary relation (in the limit of "large" objects). 
ACKNOWLEDGEMENTS. An essential part of the research was done during the visit of the first author to Uppsala, which was supported by the Faculty of Natural Sciences of Uppsala University. The financial support and hospitality of Uppsala University are gratefully acknowledged. For the second author the research was partially supported by the Swedish Research Council.

\section{Category of partitioned binary relations}

We denote by $\mathbf{N}$ and $\mathrm{N}_{0}$ the sets of all positive and non-negative integers, respectively.

\subsection{Partitioned binary relations}

Let $X$ and $Y$ be finite sets. A partitioned binary relation (PBR) on $(X, Y)$ is a binary relation $\alpha$ on the disjoint union of $X$ and $Y$. The sets $X$ and $Y$ are called the domain and the codomain of $\alpha$ and denoted by $\operatorname{Dom}(\alpha)$ and $\operatorname{Codom}(\alpha)$, respectively. Clearly, the number of partitioned binary relations on $(X, Y)$ equals $2^{(|X|+|Y|)^{2}}$.

Sometimes it might happen that $X \cap Y \neq \varnothing$, or even $X=Y$. In this case to distinguish between elements of the domain and the codomain, we write $a^{(d)}$ or $a^{(c)}$ for elements of $\operatorname{Dom}(\alpha)$ and $\operatorname{Codom}(\alpha)$, respectively.

A PBR $\alpha$ on $(X, Y)$ will be depicted as a directed graph drawn within a rectangular frame, with elements of $X$ and $Y$ represented by vertexes positioned on the right and left hand sides of the frame, respectively. The fact that $\alpha$ contains an edge $(a, b) \in(X \sqcup Y)^{2}$ will be written $(a, b) \in \alpha$ and visualized by an arrow from $a$ to $b$ on the graph. We will call $a$ and $b$ elements while $(a, b)$ will be called an edge. An example of a partitioned binary relation from $X=\left\{x_{1}, x_{2}, x_{3}, x_{4}\right\}$ to $Y=\left\{y_{1}, y_{2}, y_{3}, y_{4}, y_{5}, y_{6}, y_{7}, y_{8}, y_{9}\right\}$ is shown in Figure 1. One can and we will use diagrams interchangeably with the set theoretic approach to PBRs.

\subsection{Composition of partitioned binary relations}

In this subsection we define composition of PBRs in a categorical sense. That is, given a PBR $\alpha$ on $(X, Y)$ and a PBR $\beta$ on $(Y, Z)$, we define their composition $\beta \circ \alpha$, which will be a PBR on $(X, Z)$.

It will be convenient to start slightly more generally. Let $\aleph=\left(\alpha_{1}, \alpha_{2}\right.$, $\left.\alpha_{3}, \ldots, \alpha_{k}\right)$ be a composable sequence of PBRs in the above sense, that is $\operatorname{Codom}\left(\alpha_{i}\right)=\operatorname{Dom}\left(\alpha_{i+1}\right)$ for all $i=1,2, \ldots, k-1$. Set $X_{i}:=\operatorname{Dom}\left(\alpha_{i}\right)$ for $i=1,2, \ldots, k, X_{k+1}:=\operatorname{Codom}\left(\alpha_{k}\right)$, and $X_{\amalg}:=\coprod_{i=1}^{k+1} X_{i}$. A sequence $\xi=\left(a_{1}, b_{1}\right),\left(a_{2}, b_{2}\right), \ldots,\left(a_{m}, b_{m}\right)$ of edges taken from the PBRs in $\aleph$ is called $\aleph$-connected provided that 


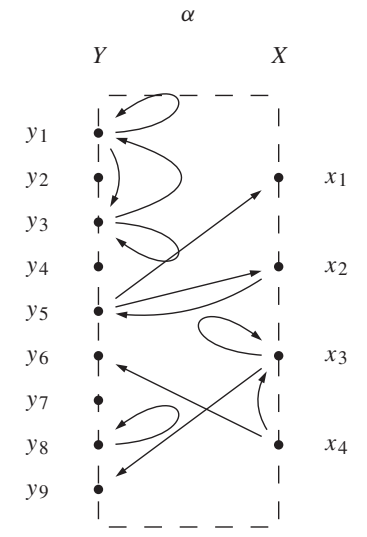

FIGURE 1. A partitioned binary relation on $(X, Y)$

(I) no two successive edges in $\xi$ are in the same PBR;

(II) for every $i=1,2, \ldots, m-1$ we have $b_{i}=a_{i+1}$ (as elements of $X_{\amalg}$ ).

We will also say that the $\aleph$-connected sequence $\xi$ connects $a_{1}$ to $b_{m}$. Note that on every step $i$ the element $b_{i}$ defines the PBR $\alpha_{j}$ containing $\left(a_{i+1}, b_{i+1}\right)$ uniquely due to condition (II). Note also that in the case $k=1$, we necessarily have $m=1$.

Let $\alpha$ be a PBR on $(X, Y)$ and $\beta$ be a PBR on $(Y, Z)$. We define the composition $\beta \circ \alpha$ as the PBR on $(X, Z)$ such that for every $a, b \in X \amalg Z$ the PBR $\beta \circ \alpha$ contains $(a, b)$ if and only if there exists an $(\alpha, \beta)$-connected sequence connecting $a$ to $b$. An example of composition of two PBRs is shown in Figure 2.
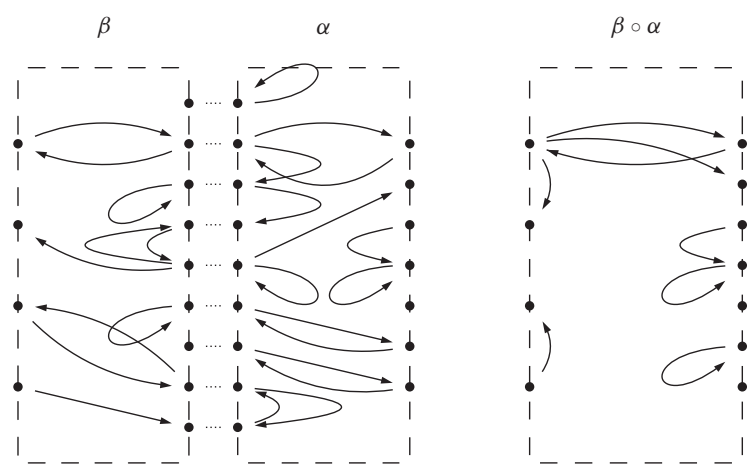

FIGURE 2. Composition of partitioned binary relations 


\subsection{Category of partitioned binary relations}

A principal observation is the following:

Proposition 2.1. Composition o defined above is associative.

Proof. Let $\alpha$ be a PBR on $(X, Y), \beta$ be a PBR on $(Y, Z)$, and $\gamma$ be a PBR on $(Z, U)$. Set $\aleph:=(\alpha, \beta, \gamma), \xi:=\beta \circ \alpha$ and $\zeta:=\gamma \circ \beta$. To prove our theorem we have to check that $(a, b) \in \gamma \circ \xi$ implies $(a, b) \in \zeta \circ \alpha$ for every $(a, b) \in(X \amalg U)^{2}$ and vice versa. We prove the first claim, the second one is proved similarly.

Let $(a, b) \in \gamma \circ \xi$ for some $(a, b) \in(X \amalg U)^{2}$. Then there is a $(\xi, \gamma)$ connected sequence $\left(a_{1}, b_{1}\right),\left(a_{2}, b_{2}\right), \ldots,\left(a_{k}, b_{k}\right)$ connecting $a$ to $b$. From this $(\xi, \gamma)$-connected sequence create a new sequence of edges by replacing every edge $\left(a_{i}, b_{i}\right) \in \xi$ in this sequence by an $(\alpha, \beta)$-connected sequence connecting $a_{i}$ to $b_{i}$ (such a sequence exists by definition of composition, but it is not necessarily unique). By construction, the obtained sequence will be ふ-connected.

Consider now all maximal consecutive subsequences of this sequence, containing only edges from $\beta$ and $\gamma$. By maximality, each such subsequences is both preceded and followed by an edge from $\alpha$, if any. From the $\aleph$-connectedness of the original sequence it follows that any such subsequence is a $(\beta, \gamma)$ connected sequence connecting its first element to its last element. Construct a new sequence by replacing each such maximal $(\beta, \gamma)$-connected subsequence by the pair of elements which this subsequence connects. This pair of elements gives an edge in $\zeta$ by definition. As a result, we obtain an $(\alpha, \zeta)$-connected sequence connecting $a$ to $b$. Hence $(a, b) \in \zeta \circ \alpha$. The claim follows.

For a finite set $X$ define the PBR $\varepsilon_{X}$ on $(X, X)$ as the one containing all edges $\left(x^{(d)}, x^{(c)}\right)$ and $\left(x^{(c)}, x^{(d)}\right)$ for all $x \in X$. The diagram of the PBR $\varepsilon_{X}$ is shown in Figure 3.

Proposition 2.2. The $\mathrm{PBR} \varepsilon_{X}$ is the identity morphism for $X$ with respect to $\circ$, that is $\varepsilon_{X} \circ \alpha=\alpha$ for any $\mathrm{PBR} \alpha$ on $(Y, X)$, and $\beta \circ \varepsilon_{X}=\beta$ for any $\mathrm{PBR}$ $\beta$ on $(X, Y)$.

Proof. This is a straightforward computation.

Adding all loops to the PBR $\varepsilon_{X}$ one obtains the idempotent $\mathrm{PBR} \bar{\varepsilon}_{X}$ (see Figure 3). Deleting all right arrows from the $\operatorname{PBR} \varepsilon_{X}$ one obtains the idempotent PBR $\hat{\varepsilon}_{X}$ (see Figure 3 ). The PBRs $\bar{\varepsilon}_{X}$ and $\hat{\varepsilon}_{X}$ will appear as identity morphisms for certain categorical substructures later on.

Define the category $\mathfrak{B B}$ of partitioned binary relations in the following way. Firstly: objects of $\mathfrak{B} \mathfrak{B}$ are finite sets; for $X, Y \in \mathfrak{B B}$ the morphism set $\mathfrak{B} \mathfrak{B}(X, Y)$ is the set of all PBRs on $(X, Y)$; the composition $\mathfrak{B} \mathfrak{B}(Y, Z) \times$ 

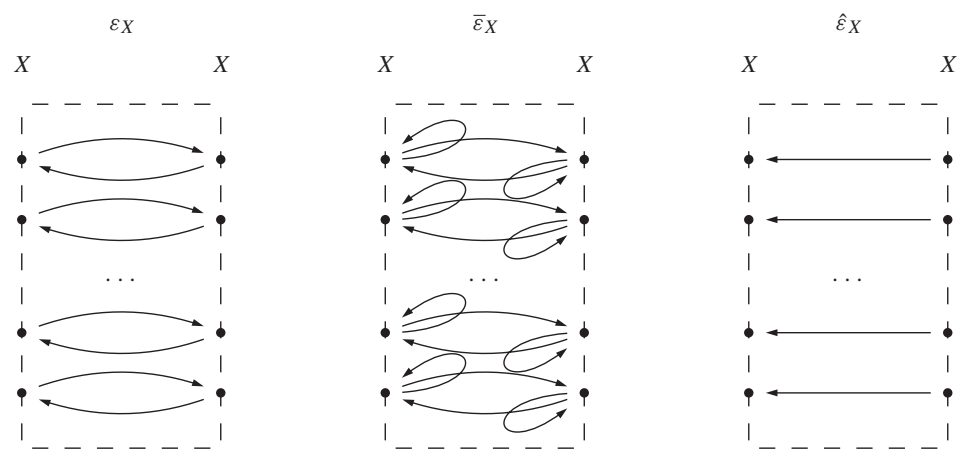

FIGURE 3. The partitioned binary relations $\varepsilon_{X}, \bar{\varepsilon}_{X}$ and $\hat{\varepsilon}_{X}$

$\mathfrak{B} \mathfrak{B}(X, Y) \rightarrow \mathfrak{B} \mathfrak{B}(X, Z)$ is given by $\circ$; for $X \in \mathfrak{B} \mathfrak{B}$ the identity morphism for $X$ is $\varepsilon_{X}$. Then, from Propositions 2.1 and 2.2 we obtain:

THEOREM 2.3. The construct $\mathfrak{B B}$ above is a category.

\subsection{Tensor product and duality}

The category $\mathfrak{B} \mathfrak{B}$ has a natural monoidal structure in which the tensor product is given on objects by the disjoint union and on morphisms by drawing diagrams next to each other as shown in Figure 4.
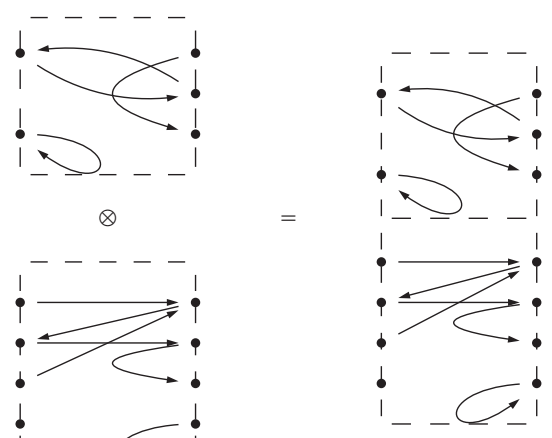

FIGURE 4. Tensor product

The category $\mathfrak{B B}$ has a natural involution (that is a contravariant object preserving involutive anti-automorphism), which we will denote by $\star$, given by taking the mirror image of the diagram with respect to a vertical mirror as shown in Figure 5. 

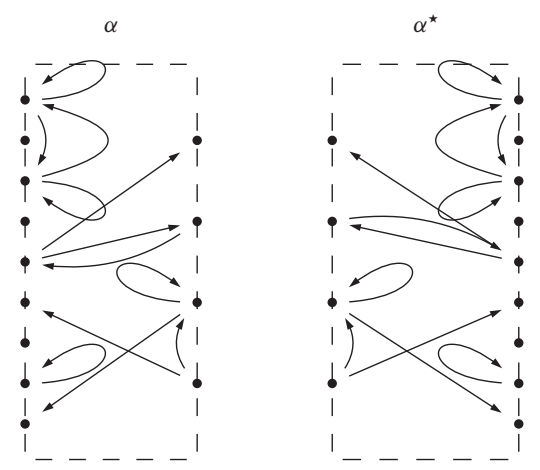

FIGURE 5. Anti-automorphism $\star$

\section{Some substructures of $\mathfrak{A B}$}

\subsection{Binary relations, first inclusion}

Consider the category $\mathfrak{B}$ of binary relations between finite sets (confer [23]). Objects of $\mathfrak{B}$ are finite sets. For $X, Y \in \mathfrak{B}$, the set $\mathfrak{B}(X, Y)$ is the set of all binary relations from $X$ to $Y$. A binary relation from $X$ to $Y$ is a subset of $X \times Y$. Such a binary relation can be viewed as a boolean matrix whose columns are indexed by elements of $X$ and rows are indexed by elements of $Y$. We shall treat the two realizations as interchangeable. Composition of binary relations may then be lifted from the usual boolean multiplication of boolean matrices (see, e.g. [23]). The identity morphism for $X$ is the equality relation (it is given by the identity matrix with respect to the same ordering of the two copies of $X$ ). The category $\mathfrak{B}$ has a natural involution $\bowtie$ given by matrix transposition.

Each binary relation from $X$ to $Y$ is a partitioned binary relation from $X$ to $Y$, in other words, $\mathfrak{B}(X, Y) \subset \mathfrak{B B}(X, Y)$. For an example, see the left element in Figure 6. It is straightforward to check that this inclusion respects composition. We will denote this inclusion by $\Phi_{1}$. Note that $\Phi_{1}$ is not a functor as it does not send the identity binary relation to the identity partitioned binary relation.

Note that $\mathfrak{B}$ has several classical subcategories, in particular,

(i) the subcategory of all maps;

(ii) the subcategory of all injective maps;

(iii) the subcategory of all partial injective maps;

(iv) the subcategory of all surjective maps;

(v) the subcategory of all partial surjective maps. 
We refer the reader to [15] for details on categories (iii) and (v). Using $\Phi_{1}$ we obtain inclusions of all these categories into $\mathfrak{B B}$ by restriction.

The image $\Phi_{1}(\mathfrak{B})$ can also be understood as an idempotent subcategory of $\mathfrak{B} \mathfrak{B}$. Let $\mathscr{C}$ be a category and $\mathbf{e}=\left(e_{X}\right)_{X \in \mathscr{C}}$ a fixed collection of idempotent endomorphisms such that $e_{X} \in \mathscr{C}(X, X)$. An e-subcategory $\mathscr{D}$ of $\mathscr{C}$ is a category such that

- objects of $\mathscr{D}$ form a subclass of objects of $\mathscr{C}$;

- $\mathscr{D}(X, Y) \subset \mathscr{C}(X, Y)$ for any $X, Y \in \mathscr{D}$;

- the multiplication in $\mathscr{D}$ is obtained from the one in $\mathscr{C}$ by restriction;

- for any $X \in \mathscr{D}$ the morphism $e_{X}$ is the corresponding identity morphism for $X$.

Among all e-subcategories of $\mathscr{C}$ there is the unique maximum one with respect to inclusions. This category is denoted by $\mathscr{C}_{\mathbf{e}}$, it has the same objects as $\mathscr{C}$ and for $X, Y \in \mathscr{C}$ we have

$$
\mathscr{C}_{\mathbf{e}}(X, Y)=e_{Y} \mathscr{C}(X, Y) e_{X}
$$

Remark 3.1. Similarly to [1, Section 5] one shows that the category of $\mathscr{C}_{\mathbf{e}^{-}}$ representations over some field $\mathrm{k}$ (that is functors from $\mathscr{C}_{\mathbf{e}}$ to $\mathrm{k}$-vector spaces) fully embeds into the category of $\mathscr{C}$-representations.

For $X \in \mathfrak{B}$ recall the idempotent PBR $\hat{\varepsilon}_{X}$ defined in Subsection 2.3 (see Figure 3). The PBR $\hat{\varepsilon}_{X}$ is the image of the identity relation on $X$ under $\Phi_{1}$.

Proposition 3.2. For $\mathbf{e}:=\left(\hat{\varepsilon}_{X}\right)_{X \in \mathfrak{B}}$ we have $\Phi_{1}(\mathfrak{B})=\mathfrak{B}_{\mathfrak{e}}$.

Proof. We have to check that for any $X, Y \in \mathfrak{B B}$ and $\alpha \in \mathfrak{B} \mathfrak{B}(X, Y)$ the following is true: $\alpha \in \Phi_{1}(\mathfrak{B})$ if and only if $\alpha=\hat{\varepsilon}_{Y} \circ \beta \circ \hat{\varepsilon}_{X}$ for some $\beta \in \mathfrak{B} \mathfrak{B}(X, Y)$.

If $\alpha \in \Phi_{1}(\mathfrak{B})$, then $\hat{\varepsilon}_{Y} \circ \alpha \circ \hat{\varepsilon}_{X}=\alpha$. On the other hand, let $\beta \in \mathfrak{B} \mathfrak{B}(X, Y)$, $\alpha=\hat{\varepsilon}_{Y} \circ \beta \circ \hat{\varepsilon}_{X}$ and $(a, b)$ be an edge of $\alpha$. Let $\left(a_{1}, b_{1}\right), \ldots,\left(a_{m}, b_{m}\right)$ be an $\left(\hat{\varepsilon}_{X}, \beta, \hat{\varepsilon}_{Y}\right)$-connected sequence connecting $a$ to $b$. From the definition of $\hat{\varepsilon}_{Y}$ it follows that $\left(a_{1}, b_{1}\right)$ is an edge from $\hat{\varepsilon}_{X}$. Similarly, $\left(a_{m}, b_{m}\right)$ is an edge from $\hat{\varepsilon}_{Y}$. This implies $a \in X$ and $b \in Y$. The claim follows.

\subsection{Binary relations, second inclusion}

With each binary relation $\theta$ from $X$ to $Y$ we associate a partitioned binary relation $\Phi_{2}(\theta)$ on $(X, Y)$ in the following way: $\Phi_{2}(\theta):=\Phi_{1}(\theta) \cup \Phi_{1}\left(\theta^{\bowtie}\right)$. The effect of $\Phi_{2}$ on binary relations is illustrated in Figure 6.

Proposition 3.3. The map $\Phi_{2}$ gives rise to a faithful functor from $\mathfrak{B}$ to $\mathfrak{B} \mathfrak{B}$.

Proof. A proof will be given in Remark 5.3. 

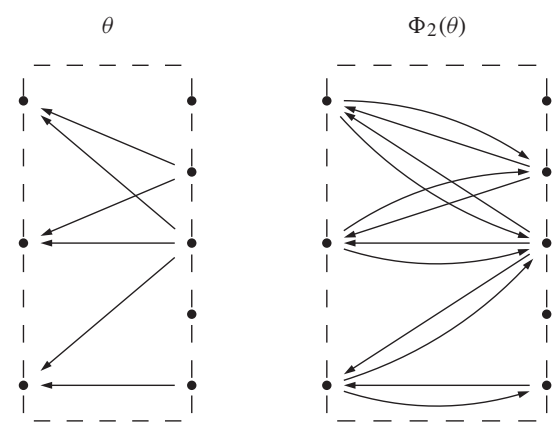

FIGURE 6. Injection $\Phi_{2}$

Similarly to Subsection 3.1, using $\Phi_{2}$ we realize categories of various types of maps as subcategories of $\mathfrak{B B}$.

\subsection{Partition category}

Denote by $\mathfrak{P}$ the partition category, defined as follows (see [16]): Objects of $\mathfrak{B}$ are finite sets. For $X, Y \in \mathfrak{R}$ the set $\mathfrak{B}(X, Y)$ is the set of all partitions of $X \amalg Y$ into a disjoint union of subsets (called parts). For $\alpha \in \mathfrak{B}(X, Y)$ and $\beta \in \mathfrak{B}(Y, Z)$ the composition $\beta \circ \alpha$ is defined as the unique partition in $\mathfrak{B}(X, Z)$ such that for any $a, b \in X \amalg Z$ the elements $a$ and $b$ belong to the same part of the partition $\beta \circ \alpha$ if and only if for some $k \in \mathrm{N}_{0}$ there is a sequence $a=a_{0}, a_{1}, \ldots, a_{k}=b$ of elements from $X \sqcup Y \amalg Z$ such that for every $i=0,1, \ldots, k-1$ the elements $a_{i}$ and $a_{i+1}$ belong to the same part of either $\alpha$ or $\beta$. The identity morphism $\pi_{X}$ of $\mathfrak{B}(X, X)$ is the partition of $X \sqcup X=X \cup X^{\prime}$, where $X^{\prime}:=\left\{x^{\prime}, x \in X\right\}$, consisting of parts $\left\{x, x^{\prime}\right\}, x \in X$.

For $X \in \mathfrak{P}$ set $\Psi(X)=X \in \mathfrak{B} \mathfrak{B}$. For $\alpha \in \mathfrak{B}(X, Y)$ denote by $\Psi(\alpha)$ the unique PBR in $\mathfrak{B B}(X, Y)$ such that for every $a, b \in X \amalg Y$ we have $(a, b) \in \Psi(\alpha)$ if and only if $a$ and $b$ belong to the same part of $\alpha$. Alternatively, we can say that the binary relation $\Psi(\alpha)$ is obtained by considering the partition $\alpha$ of $X \amalg Y$ as an equivalence relation on $X \amalg Y$. Note that $\Psi\left(\pi_{X}\right)=\bar{\varepsilon}_{X}$.

A partition is usually drawn as a diagram similarly to a diagram of PBR. Elements of the diagram are connected such that the connected components correspond to parts of the partition (note that a diagram of a partition is not uniquely defined). An example of how $\Psi$ works is given in Figure 7 (note the use of double arrows there to simplify the picture). It is straightforward to verify that for any $\alpha \in \mathfrak{B}(X, Y)$ and $\beta \in \mathfrak{B}(Y, Z)$ we have $\Psi(\beta \circ \alpha)=\Psi(\beta) \circ \Psi(\alpha)$.

Note that $\Psi$ is a not a functor as it does not map identity morphisms to identity morphisms. The image of $\Psi$ does not coincide with the idempotent subcategory of $\mathfrak{B} \mathfrak{B}$ generated by $\mathbf{e}=\left\{\bar{\varepsilon}_{X}, X \in \mathfrak{B} \mathfrak{B}\right\}$. The latter idempotent subcategory is larger. One can readily see that the subset of reflexive, transitive 

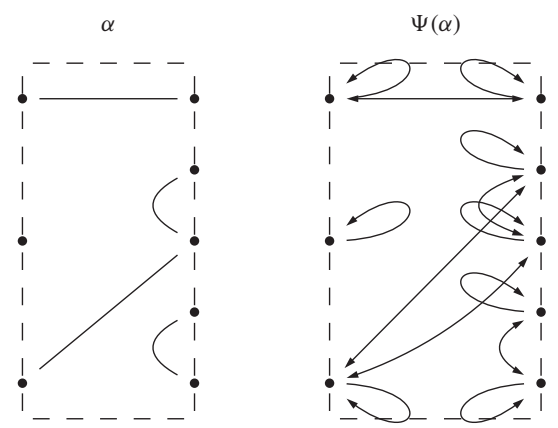

FIGURE 7. Inclusion $\Psi$

relations in $\mathfrak{P B}$ is closed under composition, and that this is $\mathfrak{P B}_{\mathrm{e}}$.

Partition category contains many classical subcategories, for example, Brauer category ([3]), partial (alias rook) Brauer category ([20]) and TemperleyLieb category ([26]). The map $\Psi$ embeds them into $\mathfrak{B} \mathfrak{B}$ by restriction.

\section{Deformation}

In this section we establish existence of a 1-parameter deformation of the category $\mathfrak{B B}$.

\subsection{Frothy elements, edges and alternating cycles}

Let $\aleph=\left(\alpha_{1}, \alpha_{2}, \alpha_{3}, \ldots, \alpha_{k}\right)$ be a composable sequence of PBRs (see Subsection 2.2). Let $X_{i}, i=1,2, \ldots, k+1$ be as in Subsection 2.2. An edge $(a, b) \in \alpha_{i}, i=1,2, \ldots, k$, is said to be $\aleph$-frothy provided that it does not occur in any $\aleph$-connected sequence connecting two (not necessarily distinct) elements from $X_{1} \amalg X_{k+1}$. For example, in the case $k=2$ shown in Figure 8 all frothy edges are drawn doubled.

An $\aleph$-connected sequence $\left(a_{1}, b_{1}\right),\left(a_{2}, b_{2}\right), \ldots,\left(a_{m}, b_{m}\right)$, where $m \in\{2$, $3,4, \ldots\}$, is called an $\aleph$-frothy cycle provided that the following conditions are satisfied:

(III) $a_{1}=b_{m}$ as elements of $X_{\mathrm{U}}$;

(IV) $\left(a_{1}, b_{1}\right)$ and $\left(a_{m}, b_{m}\right)$ come from different PBRs;

(V) all edges $\left(a_{i}, b_{i}\right), i=1,2, \ldots, m$, are $\aleph$-frothy.

Directly from the definition we have that a cyclic permutation of an $\aleph$-frothy cycle is again an $\aleph$-frothy cycle (note here importance of condition (IV) to guarantee preservation of condition (I)). We will call two $\aleph$-frothy cycles naïvely equivalent if they can be obtained from each other by a cyclic permutation. In 


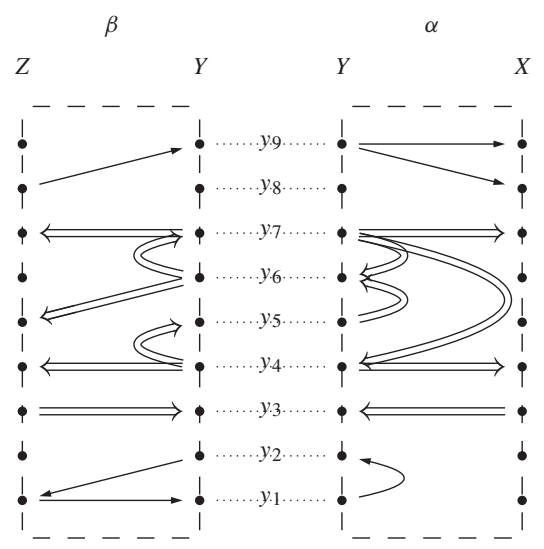

FIGURE 8. Example with $(\alpha, \beta)$-frothy edges drawn doubled

what follows we will call a naïve equivalence class of $\aleph$-frothy cycles simply a frothy cycle (if $\aleph$ is clear from the context).

Two frothy cycles are called elementary-equivalent provided that they contain a common edge and in both cycles this edge appears as an edge of the same PBR (note that the relation of elementary-equivalence is both symmetric and reflexive but not transitive in general). In the example shown in Figure 8 the two frothy cycles $\left(y_{6}, y_{7}\right),\left(y_{7}, y_{6}\right)$ and $\left(y_{4}, y_{5}\right),\left(y_{5}, y_{6}\right),\left(y_{6}, y_{7}\right),\left(y_{7}, y_{4}\right)$ are elementary-equivalent. Finally, two frothy cycles $\xi$ and $\zeta$ are called equivalent provided that there is a sequence $\xi=\xi_{0}, \xi_{1}, \ldots, \xi_{k}=\zeta$ of frothy cycles for some $k \in \mathrm{N}$ such that every pair of consecutive frothy cycles in this sequence is elementary-equivalent. This is the minimum equivalence relations containing the relation of elementary-equivalence.

Write $M_{\aleph}$ for the set of equivalence classes of $\aleph$-frothy cycles; and define $\mathfrak{f}(\aleph)=\left|M_{\aleph}\right|$. By definition, every frothy edge appears in at most one equivalence class of frothy cycles, which implies that $\mathfrak{f}(\aleph)$ is finite. In the example shown in Figure 8 we have $\mathfrak{f}((\alpha, \beta))=1$.

Proposition 4.1. Let $\alpha \in \mathfrak{B B}(X, Y), \beta \in \mathfrak{B} \mathfrak{B}(Y, Z)$ and $\gamma \in \mathfrak{B} \mathfrak{B}(Z, U)$. Then

$$
\mathfrak{f}((\beta \circ \alpha, \gamma))+\mathfrak{f}((\alpha, \beta))=\mathfrak{f}((\alpha, \beta, \gamma))=\mathfrak{f}((\alpha, \gamma \circ \beta))+\mathfrak{f}((\beta, \gamma)))
$$

Proof. We prove the left equality. The right equality then follows applying the involution $\star$. Set $\aleph:=(\alpha, \beta, \gamma)$. Then let $M_{\alpha \beta} \subset M_{\aleph}$ be the subset of equivalence classes of $\aleph$-frothy cycles satisfying the condition that every frothy cycle in the class contains only edges from $\alpha$ and $\beta$. Define $M_{\gamma}$ as the complement, so that $M_{\aleph}=M_{\alpha \beta} \sqcup M_{\gamma}$. It is easy to see that $M_{\alpha \beta}$ can be al- 
ternatively described as the set of equivalence classes containing an $\aleph$-frothy cycle all edges of which are $(\alpha, \beta)$-frothy and hence $\left|M_{\alpha \beta}\right|=\mathfrak{f}((\alpha, \beta))$.

It remains to show that $\left|M_{\gamma}\right|=\mathfrak{f}((\beta \circ \alpha, \gamma)):=\left|M_{(\beta \circ \alpha, \gamma)}\right|$. For this it is enough to establish a bijection $F: M_{(\beta \circ \alpha, \gamma)} \rightarrow M_{\gamma}$. Note that an $\aleph$-frothy cycle belonging to a class in $M_{\gamma}$ may contain no edges from $\gamma$. However, in this case it contains at least one edge from $\alpha$ or $\beta$, which is not $(\alpha, \beta)$-frothy (since there must be another cycle in its class that passes via $\gamma$ ).

We now construct $F$. Given a $(\beta \circ \alpha, \gamma)$-frothy cycle $\omega$, we substitute every $\beta \circ \alpha$-edge $(a, b)$ in $\omega$ by an $(\alpha, \beta)$-connected sequence connecting $a$ to $b$. The obtained sequence $\left(a_{1}, a_{2}\right), \ldots,\left(a_{k}, b_{k}\right)$ obviously satisfies (I)-(IV). We claim that it also satisfies $(\mathrm{V})$, that is that all $\left(a_{i}, b_{i}\right)$ are $\aleph$-frothy. Since equivalence classes contain naïve equivalence classes, it is enough to show that $\left(a_{1}, b_{1}\right)$ is $\aleph$-frothy. Assume not, and let $\omega_{1},\left(a_{1}, b_{1}\right), \omega_{2}$ be an $\aleph$-connected sequence connecting two elements of $X \amalg U$ (here $\omega_{1}$ and $\omega_{2}$ are two $\aleph$-connected sequences). Then the sequence

$$
\xi:=\omega_{1},\left(a_{1}, b_{1}\right),\left(a_{2}, b_{2}\right), \ldots,\left(a_{k}, b_{k}\right),\left(a_{1}, b_{1}\right), \omega_{2}
$$

is again $\aleph$-connected connecting the same two elements of $X \amalg U$. By definition, the original $(\beta \circ \alpha, \gamma)$-frothy cycle $\omega$ contained at least one edge from $\gamma$, say $(s, t)$. By construction, this edge appears in $\xi$. Applying to $\xi$ the procedure described in the proof of Proposition 2.1, we obtain a $(\beta \circ \alpha, \gamma)$ connected sequence which connects two elements from $X \amalg U$ and contains $(s, t)$. This means that $(s, t)$ is not $(\beta \circ \alpha, \gamma)$-frothy, a contradiction. As the result, $\left(a_{1}, a_{2}\right), \ldots,\left(a_{k}, b_{k}\right)$ is an $\aleph$-frothy cycle. It is of the second type as it contains an edge from $\gamma$. Clearly, equivalent $(\beta \circ \alpha, \gamma)$-frothy cycles are mapped to equivalent $\aleph$-frothy cycles and hence we obtain a map from $M_{(\beta \circ \alpha, \gamma)}$ to $M_{\gamma}$.

Now given an equivalence class in $M_{\gamma}$, choose a representative $\omega$, containing some edge from $\gamma$. Using the naïve equivalence, we may assume that the first edge in $\omega$ is from $\gamma$. Substitute in $\omega$ every maximal subsequence of consecutive edges from $\alpha$ and $\beta$ by the pair of elements which this sequence connects. The result will be an $(\beta \circ \alpha, \gamma)$-connected cycle and, using the arguments as in the previous paragraph, one shows that this cycle is frothy. For this procedure to define a map from $M_{\gamma}$ to $M_{(\beta \circ \alpha, \gamma)}$ we thus are left to check that equivalent $\aleph$-frothy cycles are mapped to equivalent $(\beta \circ \alpha, \gamma)$-frothy cycles. By construction, two elementary-equivalent $\aleph$-frothy cycles sharing an edge from $\gamma$ are mapped to elementary-equivalent $(\beta \circ \alpha, \gamma)$-frothy cycles. To proceed we will use the following lemma: 
Lemma 4.2. Let $\omega^{\prime}$ and $\omega^{\prime \prime}$ be equivalent $\aleph$-frothy cycles. Then there exists an $\aleph$-frothy cycle $\omega$ containing all the edges of both.

Proof. For $\aleph$-frothy cycles $\xi$ and $\xi^{\prime}$ sharing some edge $(s, t)$ we may write $\xi=\xi_{1},(s, t), \xi_{2}$ and $\xi^{\prime}=\xi_{1}^{\prime},(s, t), \xi_{2}^{\prime}$. Then denote by $\xi \square \xi^{\prime}$ the $\aleph-$ frothy cycle $\xi_{1},(s, t), \xi_{2}^{\prime}, \xi_{1}^{\prime},(s, t), \xi_{2}$. Now let $\omega^{\prime}=\omega_{1}, \omega_{2}, \ldots, \omega_{m}=\omega^{\prime \prime}$ be a sequence of $\aleph$-frothy cycles such that every pair of consecutive cycles is elementary-equivalent, with a given shared edge; and take

$$
\omega:=\left(\cdots\left(\left(\omega_{1} \square \omega_{2}\right) \square \omega_{3}\right) \square \cdots\right) \square \omega_{m} .
$$

Let $\omega^{\prime}$ and $\omega^{\prime \prime}$ be equivalent $\aleph$-frothy cycles, each containing some edge from $\gamma$, and $\omega$ be an $\aleph$-frothy cycle given by Lemma 4.2. Then $\omega^{\prime}$ and $\omega$ are elementary-equivalent, as are $\omega^{\prime \prime}$ and $\omega$. By the paragraph preceding Lemma 4.2, we have that $\omega^{\prime}$ and $\omega$ are mapped to elementary-equivalent $(\beta \circ \alpha, \gamma)$-frothy cycles, as are $\omega^{\prime \prime}$ and $\omega$. It follows that the images of $\omega^{\prime}$ and $\omega^{\prime \prime}$ are equivalent, giving us a well-defined map from $M_{\gamma}$ to $M_{(\beta \circ \alpha, \gamma)}$.

From their constructions it follows directly that the maps between $M_{\gamma}$ and $M_{(\beta \circ \alpha, \gamma)}$ are mutually inverse bijections. This completes the proof.

\subsection{Deformed category}

We consider $\mathrm{N}_{0}$ as an additive monoid in the natural way. Consider the category $\overline{\mathfrak{A B}}$ defined as follows: objects of $\overline{\mathfrak{P B}}$ are the same as objects of $\mathfrak{B B}$; for $X, Y \in \overline{\mathfrak{P B}}$ the morphism set $\overline{\mathfrak{P B}}(X, Y)$ equals $\mathfrak{B} \mathfrak{B}(X, Y) \times \mathrm{N}_{0}$; for $(\alpha, k) \in$ $\overline{\mathfrak{B S}}(X, Y)$ and $(\beta, m) \in \overline{\mathfrak{P B}}(Y, Z)$ set

$$
(\beta, m) \diamond(\alpha, k):=(\beta \circ \alpha, m+k+\mathfrak{f}(\alpha, \beta)) .
$$

THEOREM 4.3. The above definition makes $\overline{\mathfrak{P} B}$ into a category.

Proof. Associativity of $\diamond$ follows from Proposition 4.1. Note that the identity morphism $\varepsilon_{X}$ in $\mathfrak{B B}(X, X)$ does not have any edges connecting two elements of the codomain. This implies that for any $\alpha \in \mathfrak{B} \mathfrak{B}(X, Y)$ we have $\mathfrak{f}\left(\varepsilon_{X}, \alpha\right)=\mathfrak{f}\left(\alpha, \varepsilon_{Y}\right)=0$. Hence $\left(\varepsilon_{X}, 0\right)$ is the identity morphism in $\overline{\mathfrak{B} \mathfrak{B}}(X, X)$. The claim follows.

\subsection{Deformed partition category via restriction}

Recall, from [16], that the category $\mathfrak{P}$ admits deformation $\overline{\mathfrak{P}}$, similar to the deformation $\overline{\mathfrak{P B}}$ of $\mathfrak{B B}$. It is constructed as follows: The category $\overline{\mathfrak{P}}$ has the same objects as $\mathfrak{P}$. For $X, Y \in \overline{\mathfrak{P}}$ the set $\overline{\mathfrak{P}}(X, Y)$ equals $\mathfrak{P}(X, Y) \times \mathrm{N}_{0}$ and the multiplication in $\overline{\mathfrak{P}}(X, Y)$ is given for $(\alpha, k) \in \overline{\mathfrak{P}}(X, Y)$ and $(\beta, m) \in \overline{\mathfrak{P}}(Y, Z)$ by the following:

$$
(\beta, m) \diamond(\alpha, k):=(\beta \circ \alpha, m+k+\mathfrak{p}(\alpha, \beta)),
$$


where $\mathfrak{p}(\alpha, \beta)$ is defined as follows: Denote by $Y^{\prime}$ the set of all $y \in Y$ for which there does not exist a sequence $y=a_{1}, a_{2}, a_{3}, \ldots, a_{p}$, where all $a_{i} \in$ $X \amalg Y \amalg Z, a_{p} \in X \amalg Z$, and such that every two consecutive elements in this sequence belong to the same part of either $\alpha$ or $\beta$. Introduce an equivalence relation $\sim$ on $Y^{\prime}$ as follows: $y_{1} \sim y_{2}$ for $y_{1}, y_{2} \in Y^{\prime}$ if and only if there is a sequence $y_{1}=a_{1}, a_{2}, a_{3}, \ldots, a_{p}=y_{2}$, where all $a_{i} \in Y$, such that every two consecutive elements in this sequence belong to the same part of either $\alpha$ or $\beta$. Then $\mathfrak{p}(\alpha, \beta)$ is defined as the number of equivalence classes of $\sim$. Our main observation in this subsection is the following statement which says that $\Psi$ can be lifted up to the level of deformed categories.

Proposition 4.4. Define $\bar{\Psi}: \overline{\mathfrak{P}} \rightarrow \overline{\mathfrak{B B}}$ as the identity on objects and $\bar{\Psi}((\alpha, k)):=(\Psi(\alpha), k)$ for any morphism $(\alpha, k)$. Then

$$
\bar{\Psi}((\beta, m) \diamond(\alpha, k))=\bar{\Psi}((\beta, m)) \diamond \bar{\Psi}((\alpha, k))
$$

for all composable morphisms $(\alpha, k)$ and $(\beta, m)$ in $\bar{\Re}$.

Proof. To prove this statement we need to check that for any morphisms $\alpha \in \mathfrak{P}(X, Y)$ and $\beta \in \mathfrak{P}(Y, Z)$ there is a bijection between the set $M_{1}$ of equivalence classes for the relation $\sim$ defined above and the set $M_{2}$ of equivalence classes of $(\Psi(\alpha), \Psi(\beta))$-frothy cycles.

Every $(\Psi(\alpha), \Psi(\beta))$-frothy cycle consists of edges between elements in $Y$. From the definition of $\Psi$ it follows easily that all these elements, in fact, belong to $Y^{\prime}$. Moreover, from the definition of $\sim$ it follows that all these element are $\sim$-related. Hence we can define a map from the set of $(\Psi(\alpha), \Psi(\beta))$-frothy cycles to $M_{1}$ by sending each cycle to the corresponding equivalence class of $\sim$ described above. Since $\sim$ is an equivalence relation, elementary equivalent cycles have the same image. This means that this map factors through $M_{2}$ giving us a map from $M_{2}$ to $M_{1}$.

First of all we claim that this map is surjective. Indeed, given an equivalence class $N$ of $\sim$, let $y \in N$. Then the construction of $\Psi$ implies that the edge $(y, y)$ is contained both in $\Psi(\alpha)$ and $\Psi(\beta)$. Therefore $(y, y),(y, y)$ is a $(\Psi(\alpha), \Psi(\beta))$-frothy cycle (in which the first edge is in $\Psi(\alpha)$ and the second edge is in $\Psi(\beta))$. By construction, the cycle $(y, y),(y, y)$ is mapped to $N$, which implies surjectivity.

Now we claim that our map is injective. Let $N$ be an equivalence class of $\sim$. To prove the assertion we have to show that all $(\Psi(\alpha), \Psi(\beta))$-frothy cycles mapped to $N$ are equivalent. For this it is enough to show that every $(\Psi(\alpha), \Psi(\beta))$-frothy cycle mapped to $N$ is equivalent to a $(\Psi(\alpha), \Psi(\beta))$ frothy cycle of the form $(y, y),(y, y)$ as above; and that all such $(\Psi(\alpha), \Psi(\beta))$ frothy cycles are equivalent. 
Let $\omega$ be one of the $(\Psi(\alpha), \Psi(\beta))$-frothy cycles and $(s, t)$ its first edge. Then $(s, s),(s, s), \omega$, where the first edge $(s, s)$ is considered from the same factor $(\alpha$ or $\beta)$ as the edge $(s, t)$ of $\omega$, is a $(\Psi(\alpha), \Psi(\beta))$-frothy cycle, which is elementary equivalent to $\omega$. On the other hand, the cycle $(s, s),(s, s), \omega$ is elementary equivalent to $(s, s),(s, s)$. Hence $\omega$ is equivalent to $(s, s),(s, s)$.

Now let $s, t \in N$ and $s=a_{1}, a_{2}, \ldots, a_{k}=t$ be a sequence of elements from $Y^{\prime}$ in which every pair of consecutive elements belongs to the same part of either $\alpha$ or $\beta$. Without loss of generality we may even assume that this alternates in the sense that if $a_{1}$ and $a_{2}$ belong to the same edge of $\alpha$, then $a_{2}$ and $a_{3}$ belong to the same edge of $\beta$ and so on. From the definition of $\Psi$ it follows that we have a $(\Psi(\alpha), \Psi(\beta))$-connected sequence as follows: $\left(a_{1}, a_{2}\right),\left(a_{2}, a_{3}\right), \ldots,\left(a_{k-1}, a_{k}\right)$. This yields existence of a $(\Psi(\alpha), \Psi(\beta))$ frothy cycle as follows:

$$
\begin{aligned}
\omega:=\left(a_{1}, a_{2}\right),\left(a_{2}, a_{3}\right), \ldots,\left(a_{k-1}, a_{k}\right), & \\
& \left(a_{k}, a_{k}\right),\left(a_{k}, a_{k-1}\right), \ldots,\left(a_{2}, a_{1}\right),\left(a_{1}, a_{1}\right) .
\end{aligned}
$$

Here $\left(a_{i}, a_{i-1}\right)$ and $\left(a_{i-1}, a_{i}\right)$ are considered as edges of the same factor $(\alpha$ or $\beta),\left(a_{k}, a_{k}\right)$ is considered as an edge from the factor, different from the factor containing $\left(a_{k-1}, a_{k}\right)$, and $\left(a_{1}, a_{1}\right)$ is considered as an edge from the factor, different from the factor containing $\left(a_{1}, a_{2}\right)$. The cycle $\omega$ is elementary equivalent to both $(s, s),(s, s)$ and $(t, t),(t, t)$, which implies that the latter two cycles are equivalent. This yields injectivity.

The above implies that our map is bijective and the claim of the proposition follows.

The deformation $\bar{P}$ of the partition category contains deformations of both Brauer and Temperley-Lieb categories as well as the one-parameter deformation of the partial Brauer category ([21]). We note that by a Brauer category we mean the category defined combinatorially using Brauer diagrams (this corresponds to the classical definition of [3] when the defining parameter is 1) while by a one-parameter deformation of the Brauer category we mean the category which corresponds to the classical definition of [3] for generic parameter.

The map $\bar{\Psi}$ embeds them into $\overline{\mathfrak{B} B}$ by restriction. Some diagram categories admit a two-parameter deformation, see [21], [19], [18]. However, we do not know how to realize these one in terms of the category $\overline{\mathfrak{B R}}$.

\subsection{Oriented Brauer and Temperley-Lieb categories}

For finite sets $X$ and $Y$ a PBR $\alpha \in \mathfrak{B B}(X, Y)$ is called an oriented partial Brauer diagram provided that every element $s \in X \amalg Y$ appears in at most one edge of $\alpha$. An oriented partial Brauer diagram $\alpha$ for which every element $s \in X \amalg Y$ appears in exactly one edge of $\alpha$ is called an oriented Brauer 
diagram. An example of an oriented partial Brauer diagram is given in Figure 9. One can say that an oriented (partial) Brauer diagram is obtained from a usual (partial) Brauer diagram (see [3], [20]) by choosing orientation of all chords on the latter. A (partial) Brauer diagram is obtained from an oriented (partial) Brauer diagram by forgetting the orientation.

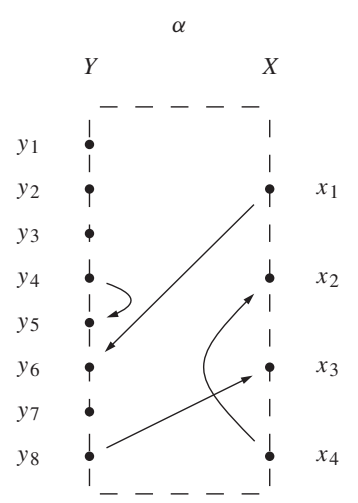

FIGURE 9. Oriented partial Brauer diagram

Lemma 4.5. Let $\alpha$ be a PBR on $(X, Y)$ and $\beta$ be a PBR on $(Y, Z)$. Assume that both $\alpha$ and $\beta$ are oriented partial Brauer diagrams. Then we have the following:

(a) The composition $\beta \circ \alpha$ is an oriented partial Brauer diagram.

(b) The number $\mathfrak{f}((\alpha, \beta))$ is the number of oriented cycles on the diagram from Figure 8.

Proof. Any element of $X$ and $Z$ appears in at most one edge of $\alpha$ or $\beta$, respectively. Any element of $Y$ appears in at most one edge of $\alpha$ and in at most one edge of $\beta$. Hence for every $s \in X \amalg Z$, there is at most one $(\alpha, \beta)$-connected sequence connecting $s$ to some element $t \in X \amalg Z$, moreover, $s \neq t$. This implies both claim (a) and the fact that every equivalence class of $(\alpha, \beta)$-frothy cycles consists of a single element. The latter implies claim (b).

The collection of all oriented partial Brauer diagrams does not give rise to a subcategory of $\mathfrak{B} \mathfrak{B}$ (or $\overline{\mathfrak{B B}}$ ) because of the absence of identity morphisms. The collection of all oriented Brauer diagrams is not even closed under composition (the composition of two oriented Brauer diagrams is only an oriented partial Brauer diagram in general). One can remedy the situation in the following way (confer [24]).

Define the category $\subseteq$ as follows: Objects of $\subseteq$ are pairs $\mathbf{X}:=\left(X_{1}, X_{2}\right)$ of finite sets such that $X_{1} \subset X_{2}$. For $\mathbf{X}, \mathbf{Y} \in \mathfrak{D}$ the set $\mathfrak{D}(\mathbf{X}, \mathbf{Y})$ consists of all 
pairs $(\alpha, k)$, where $k \in \mathrm{N}_{0}$ and $\alpha$ is an oriented Brauer diagram $\alpha$ on $\left(X_{2}, Y_{2}\right)$ such that the following condition is satisfied:

(4.3) For every edge $(a, b) \in \alpha$ we have

$$
a \in X_{1} \cup\left(Y_{2} \backslash Y_{1}\right) \text { and } b \in Y_{1} \cup\left(X_{2} \backslash X_{1}\right) \text {. }
$$

For $(\alpha, k) \in \mathfrak{D}(\mathbf{X}, \mathbf{Y})$ and $(\beta, m) \in \mathfrak{D}(\mathbf{Y}, \mathbf{Z})$ define the composition $(\beta, m) \diamond$ $(\alpha, k)$ by formula (4.2). For $\mathbf{X} \in \mathfrak{D}$ denote by $\check{\varepsilon}_{\mathbf{X}}$ the oriented Brauer diagram of the identity morphism for $\mathbf{X}$. This diagram consists of all edges $\left(x^{(d)}, x^{(c)}\right)$, $x \in X_{1}$, and $\left(x^{(c)}, x^{(d)}\right), x \in X_{2} \backslash X_{1}$, see example in Figure 10.

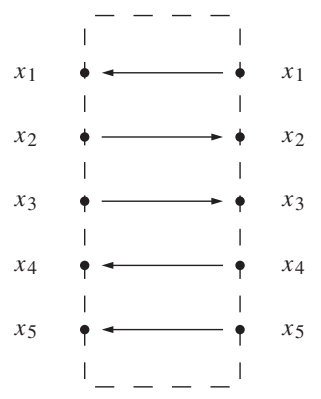

FIGURE 10. The oriented Brauer diagram $\check{\varepsilon}_{\left(\left\{x_{1}, x_{4}, x_{5}\right\},\left\{x_{1}, x_{2}, x_{3}, x_{4}, x_{5}\right\}\right)}$

Proposition 4.6. The construct $\subseteq$ above is a category, called oriented Brauer category.

Proof. For $(\alpha, k) \in \mathfrak{S}(\mathbf{X}, \mathbf{Y})$ and $(\beta, m) \in \mathfrak{D}(\mathbf{Y}, \mathbf{Z})$, from the definition of $\bigcirc$ it follows immediately that $\beta \circ \alpha$ is an oriented Brauer diagram. Now associativity is obtained from Theorem 4.3 by restriction. The fact that the $\check{\varepsilon}_{\mathbf{X}}$ 's are identity morphisms is proved by a straightforward computation.

The (standard skeleton of) classical Brauer category has a natural topological counterpart, known as the category of tangles (see e.g. [28]). The natural topological counterpart of the category $\mathfrak{D}$ is the category of oriented tangles, see [27]. The corresponding planar objects are the Temperley-Lieb and the oriented Temperley-Lieb categories. To define the oriented Temperley-Lieb category $\subseteq \mathfrak{I} \mathbb{R}$ for every finite set $X$ fix a linear order $<_{X}$ on $X$. Then the category $\mathfrak{D} \mathbb{I}$ is defined as the subcategory of $\mathfrak{D}$ with the same set of objects and containing all those morphisms $(\alpha, k)$ for which the diagram of $\alpha$ can be drawn planar (whenever the elements of the domain and the codomain are listed with respect to the fixed linear order from top to bottom). Similarly one defines the partial oriented Brauer category $\mathscr{P} \mathfrak{D}$ and the partial oriented Temperley-Lieb category $\mathscr{P} \mathfrak{N} \mathbb{I}$. 


\section{Polarized factorization}

In this section we establish a factorization of partitioned binary relations, called polarized factorization.

\subsection{Pure partitioned binary relations}

Let $X, Y \in \mathfrak{B B}$ and $\alpha \in \mathfrak{S B}(X, Y)$. The $\mathrm{PBR} \alpha$ is called pure provided that every edge in $\alpha$ consists of an element in $\operatorname{Dom}(\alpha)$ and an element in $\operatorname{Codom}(\alpha)$. For example, both PBRs $\varepsilon_{X}$ and $\hat{\varepsilon}_{X}$ are pure while the PBR $\bar{\varepsilon}_{X}$ is not pure (see Figure 3). Another example of a pure PBR is shown in Figure 11 in the middle.
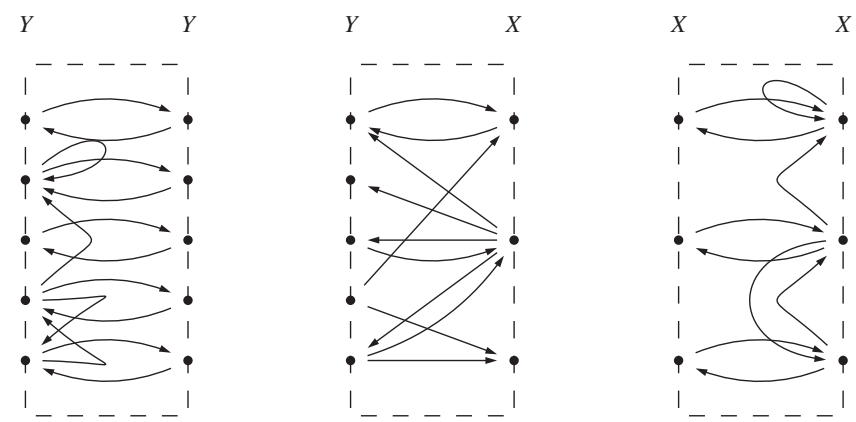

FIGURE 11. Left polarized idempotent, pure PBR and right polarized idempotent

Lemma 5.1. The composition of two composable pure $\mathrm{PBR}$ is pure. Hence, taking all pure $\mathrm{PBR}$ as morphisms defines a subcategory of $\mathfrak{B} B$ of pure $\mathrm{PBR}$, which we will denote by $\mathfrak{B R B}$.

Proof. As the $\operatorname{PBR} \varepsilon_{X}$ of the identity morphism is pure, to prove the claim we have only to check that pure PBRs are closed with respect to composition. This follows directly from definitions.

The category $\overline{\mathfrak{B} B}$ of Section 4.2 contains a subcategory $\mathfrak{P} \overline{\mathfrak{B} B}$ which has the same objects as $\overline{\mathfrak{B} B}$ and whose morphisms are all morphisms of the form $(\alpha, 0)$, where $\alpha$ is a morphism from $\mathfrak{B R B R}$. It is easy to see that no frothy cycles appear when composing two pure PBRs, and hence the categories $\mathfrak{B P B B}$ and $\mathfrak{B R B}$ are isomorphic.

The category $\mathfrak{B} \mathfrak{B} \mathfrak{B}$ admits a nice description in terms of the category $\mathfrak{B}$ of binary relations. Consider the double $\mathfrak{B}^{\ltimes}$ of the category $\mathfrak{B}$ defined as follows: Objects of $\mathfrak{B}^{\ltimes}$ are the same as objects of $\mathfrak{B}$. For $X, Y \in \mathfrak{B}^{\ltimes}$ the set $\mathfrak{B}^{\ltimes}(X, Y)$ consists of pairs $(\beta, \gamma)$, where $\beta \in \mathfrak{B}(X, Y)$ and $\gamma \in \mathfrak{B}^{\circ \mathrm{p}}(X, Y)$ (the opposite category). For $(\beta, \gamma) \in \mathfrak{B}^{\ltimes}(X, Y)$ and $\left(\beta^{\prime}, \gamma^{\prime}\right) \in \mathfrak{B}^{\ltimes}(Y, Z)$ the composition is defined as follows:

$$
\left(\beta^{\prime}, \gamma^{\prime}\right)(\beta, \gamma)=\left(\beta^{\prime} \beta, \gamma \gamma^{\prime}\right)
$$


Proposition 5.2. The categories $\mathfrak{B}^{\ltimes}$ and $\mathfrak{B} \mathfrak{B} \mathfrak{B}$ are isomorphic.

Proof. By definition, these categories have the same objects. For $\alpha \in$ $\mathfrak{P R B}(X, Y)$, where $X, Y \in \mathfrak{P R B}$, let $\beta \in \mathfrak{B}(X, Y)$ be the collection of all edges $(a, b)$ of $\alpha$ such that $a \in \operatorname{Dom}(\alpha)$ and $b \in \operatorname{Codom}(\alpha)$. Let $\gamma \in$ $\mathfrak{B}^{\mathrm{op}}(X, Y)$ be the collection of all edges $(a, b)$ of $\alpha$ such that $a \in \operatorname{Codom}(\alpha)$ and $b \in \operatorname{Dom}(\alpha)$. From the definition of pure PBRs it follows easily that the map $\alpha \mapsto(\beta, \gamma)$ is a bijection from $\mathfrak{P} \mathfrak{P B}(X, Y)$ to $\mathfrak{B}^{\ltimes}(X, Y)$. It is also easy to check that this map is compatible with compositions on both sides. The claim follows.

REMARK 5.3. Under the identification of $\mathfrak{B}^{\ltimes}$ and $\mathfrak{P} \mathfrak{P} \mathfrak{B}$ from Proposition 5.2, the "diagonal" image of $\mathfrak{B}$ in $\mathfrak{B}^{\ltimes}$ given by $\alpha \mapsto\left(\alpha, \alpha^{\bowtie}\right)$ coincides with $\Phi_{2}(\mathfrak{B})$ (see Subsection 3.2). This implies Proposition 3.3.

\subsection{Left and right polarized idempotents}

Let $X \in \mathfrak{B B}$ and $\alpha \in \mathfrak{B} \mathfrak{B}(X, X)$. The element $\alpha$ is called a left polarized idempotent provided that $\alpha$ contains all edges from $\varepsilon_{X}$ and any other edge of $\alpha$ has the form $(a, b)$, where $a, b \in \operatorname{Codom}(\alpha)$. Define a right polarized idempotent similarly using $\operatorname{Dom}(\alpha)$. It is easy to see that every left (right) polarized idempotent is indeed an idempotent. In particular, the identity morphism $\varepsilon_{X}$ is both, left and right, polarized. An example of a left polarized idempotent is given in Figure 11 on the left. An example of a right polarized idempotent is given in Figure 11 on the right. We denote by $P I(X, l)$ and $P I(X, r)$ the sets of left and right polarized idempotents in $\mathfrak{R} \mathfrak{B}(X, X)$, respectively.

Lemma 5.4. Both $P I(X, l)$ and $P I(X, r)$ are submonoids of $\mathfrak{B} \mathfrak{B}(X, X)$ isomorphic to the commutative band (semilattice) $(\mathfrak{B}(X, X), \cup)$. In particular, we have $|P I(X, l)|=|P I(X, r)|=2^{|X|^{2}}$.

PROOF. Straightforward computation.

\subsection{Polarized factorization of partitioned binary relations}

Let $X, Y \in \mathfrak{P B}$ and $\alpha \in \mathfrak{P B}(X, Y)$. Define $\gamma_{\alpha}$ as the pure PBR on $(X, Y)$ consisting of all edges from $\alpha$, which contain an element in $\operatorname{Dom}(\alpha)$ and an element in $\operatorname{Codom}(\alpha)$. Define $\beta_{\alpha}$ as the left polarized idempotent in $\mathfrak{R B}(Y, Y)$ such that for every edge $(a, b)$, where $a, b \in Y=\operatorname{Codom}(\alpha)$ we have $(a, b) \in$ $\alpha$ if and only if $(a, b) \in \beta_{\alpha}$. Define $\delta_{\alpha}$ as the right polarized idempotent in $\mathfrak{P B}(X, X)$ such that for every edge $(a, b)$, where $a, b \in X=\operatorname{Dom}(\alpha)$ we have $(a, b) \in \alpha$ if and only if $(a, b) \in \delta_{\alpha}$.

The main result of this section is the following statement establishing polarized factorization of partitioned binary relations. 
Theorem 5.5. Let $X, Y \in \mathfrak{B} \mathfrak{B}, \alpha \in \mathfrak{B B}(X, Y)$ and $\beta_{\alpha}, \gamma_{\alpha}$ and $\delta_{\alpha}$ be as defined above. Then $\alpha=\beta_{\alpha} \circ \gamma_{\alpha} \circ \delta_{\alpha}$ is the unique factorization of $\alpha$ into a product of a left polarized idempotent, a pure PBR and a right polarized idempotent.

Proof. That $\alpha=\beta_{\alpha} \circ \gamma_{\alpha} \circ \delta_{\alpha}$ is checked by a straightforward computation, proving existence. Having established existence, uniqueness is proved by a counting argument. Indeed, we have $|\mathfrak{B B B}(X, Y)|=2^{(|X|+|Y|)^{2}}$. At the same time, the number of left polarized idempotents in $\mathfrak{B} \mathfrak{B}(Y, Y)$ equals $2^{|Y|^{2}}$, the number of right polarized idempotents in $\mathfrak{B B}(X, X)$ equals $2^{|X|^{2}}$ and the number of pure PBRs on $(X, Y)$ equals $2^{2|X||Y|}$. Hence the multiplication rule implies that

$$
|\mathfrak{R B}(X, Y)|=|P I(Y, l) \times \mathfrak{B} \mathfrak{R} \mathfrak{B}(X, Y) \times P I(X, r)|
$$

and the claim follows.

It is easy to see that the polarized factorization in $\mathfrak{B} \mathfrak{B}$ gives rise to a factorization in $\overline{\mathfrak{s} B}$. Theorem 5.5 shows that morphisms of the relatively complicated category $\mathfrak{B} \mathfrak{B}$ decompose canonically into a product of morphisms from the less complicated category $\mathfrak{B} \mathfrak{B B}$ and elements of some commutative bands.

\subsection{Composition of PBRs via composition of binary relations}

The polarized decomposition of PBRs motivates the following construction: For a PBR $\alpha$ consider the following subsets of $\alpha$ :

$$
\begin{aligned}
& \alpha_{11}:=\{(a, b) \in \alpha: a \in \operatorname{Dom}(\alpha), b \in \operatorname{Dom}(\alpha)\}, \\
& \alpha_{12}:=\{(a, b) \in \alpha: a \in \operatorname{Dom}(\alpha), b \in \operatorname{Codom}(\alpha)\}, \\
& \alpha_{21}:=\{(a, b) \in \alpha: a \in \operatorname{Codom}(\alpha), b \in \operatorname{Dom}(\alpha)\}, \\
& \alpha_{22}:=\{(a, b) \in \alpha: a \in \operatorname{Codom}(\alpha), b \in \operatorname{Codom}(\alpha)\} .
\end{aligned}
$$

Then $\alpha$ is a disjoint union of the $\alpha_{i j}$ 's, $i, j=1,2$. Moreover, the $\alpha_{i j}$ 's can be interpreted in terms of factors of the polarized decomposition of $\alpha$ in the obvious way (i.e. $\gamma_{\alpha}=\alpha_{12} \cup \alpha_{21}, \delta_{\alpha}=\varepsilon_{X} \cup \alpha_{11}$ and $\beta_{\alpha}=\varepsilon_{Y} \cup \alpha_{22}$ ).

Given a PBR $\beta$ composable with $\alpha$, directly from the definition of the 
product we obtain the following formulae:

$$
\begin{aligned}
& (\beta \circ \alpha)_{11}=\alpha_{11} \cup \bigcup_{i \geq 1} \alpha_{21} \circ\left(\beta_{11} \circ \alpha_{22}\right)^{i} \circ \beta_{11} \circ \alpha_{12}, \\
& (\beta \circ \alpha)_{22}=\beta_{22} \cup \bigcup_{i \geq 1} \beta_{12} \circ\left(\alpha_{22} \circ \beta_{11}\right)^{i} \circ \alpha_{22} \circ \beta_{21}, \\
& (\beta \circ \alpha)_{12}=\bigcup_{i \geq 0} \beta_{12} \circ\left(\alpha_{22} \circ \beta_{11}\right)^{i} \circ \alpha_{12}, \\
& (\beta \circ \alpha)_{21}=\bigcup_{i \geq 0} \alpha_{21} \circ\left(\beta_{11} \circ \alpha_{22}\right)^{i} \circ \beta_{21} .
\end{aligned}
$$

\subsection{On random products of $\mathrm{PBRs}$}

For a finite set $X$ denote by $\omega_{X}$ the maximum binary relation on $X$ with respect to inclusions (i.e. the full relation). Denote also by $\bar{\omega}_{X}$ the maximum PBR on $(X, X)$ with respect to inclusions. Let $A_{X}$ denote the set of all pairs $\left(\alpha, \alpha^{\prime}\right) \in \mathfrak{B}(X, X) \times \mathfrak{B}(X, X)$ such that $\alpha \circ \alpha^{\prime}=\omega_{X}$. Let $\bar{A}_{X}$ denote the set of all pairs $\left(\alpha, \alpha^{\prime}\right) \in \mathfrak{B} \mathfrak{B}(X, X) \times \mathfrak{P B}(X, X)$ such that $\alpha \circ \alpha^{\prime}=\bar{\omega}_{X}$. Recall the following classical result (see e.g. [12, Theorem 4]):

Proposition 5.6. We have:

$$
\lim _{|X| \rightarrow \infty} \frac{\left|A_{X}\right|}{|\mathfrak{B}(X, X) \times \mathfrak{B}(X, X)|}=1 .
$$

Let $A_{X}^{\prime}$ denote the set of all $\left(\alpha, \alpha^{\prime}, \alpha^{\prime \prime}\right) \in \mathfrak{B}(X, X) \times \mathfrak{B}(X, X) \times \mathfrak{B}(X, X)$ such that $\alpha \circ \alpha^{\prime} \circ \alpha^{\prime \prime}=\omega_{X}$.

COROLlary 5.7. We have:

$$
\lim _{|X| \rightarrow \infty} \frac{\left|A_{X}^{\prime}\right|}{|\mathfrak{B}(X, X) \times \mathfrak{B}(X, X) \times \mathfrak{B}(X, X)|}=1 .
$$

Proof. By Proposition 5.6, when $|X| \rightarrow \infty$ both the probability of $\alpha \circ \alpha^{\prime}=$ $\omega_{X}$ and of $\alpha^{\prime} \circ \alpha^{\prime \prime}=\omega_{X}$ tend to 1 . Hence the probability of the intersection of these events tends to 1 as well. However, if $\alpha^{\prime} \circ \alpha^{\prime \prime}=\omega_{X}$, then the Boolean matrix of $\alpha^{\prime \prime}$ cannot have zero columns. Hence, in this case $\alpha \circ \alpha^{\prime}=\omega_{X}$ implies $\alpha \circ \alpha^{\prime} \circ \alpha^{\prime \prime}=\omega_{X}$. The claim follows.

Remark 5.8. Proposition 5.6 combined with [7, Theorem 6] implies [8, Conjecture 5].

In the following statement we extend Proposition 5.6 to PBRs. 
THEOREM 5.9. We have:

$$
\lim _{|X| \rightarrow \infty} \frac{\left|\bar{A}_{X}\right|}{|\mathfrak{B B B}(X, X) \times \mathfrak{B} \mathfrak{B}(X, X)|}=1 .
$$

Proof. By Subsection 5.4, choosing a PBR $\alpha$ is equivalent to choosing four binary relations $\alpha_{i j}, i, j=1,2$. By (5.1), $\beta \circ \alpha=\bar{\omega}_{X}$ is guaranteed by the following list of conditions:

$$
\begin{aligned}
\beta_{12} \circ \alpha_{12} & =\omega_{X} ; \\
\alpha_{21} \circ \beta_{21} & =\omega_{X} ; \\
\alpha_{21} \circ \beta_{11} \circ \alpha_{12} & =\omega_{X} ; \\
\beta_{12} \circ \alpha_{22} \circ \beta_{21} & =\omega_{X} .
\end{aligned}
$$

By Proposition 5.6 and Corollary 5.7, when $|X| \rightarrow \infty$, the probability of each of these conditions tends to 1 . Hence the probability of their intersection tends to 1 as well. The claim follows.

\section{REFERENCES}

1. Auslander, M., Representation theory of Artin algebras I, Comm. Algebra 1 (1974), 177-268.

2. Bernstein, J., Frenkel, I., and Khovanov, M., A categorification of the Temperley-Lieb algebra and Schur quotients of $U\left(\mathfrak{s l}_{2}\right)$ via projective and Zuckerman functors, Selecta Math. 5 (1999), 199-241.

3. Brauer, R., On algebras which are connected with the semisimple continuous groups, Ann. of Math. 38 (1937), 857-872.

4. Cline, E., Parshall, B., and Scott, L., Generic and q-rational representation theory, Publ. Res. Inst. Math. Sci. 35 (1999), 31-90.

5. Cox, A., Martin, P., Parker, A., and Xi, C., Representation theory of towers of recollement: theory, notes, and examples, J. Algebra 302 (2006), 340-360.

6. Fitzgerald, D., and Lau, K. W., On the partition monoid and some related semigroups, Bull. Aust. Math. Soc. 83 (2011), 273-288.

7. Ganyushkin, O., and Mazorchuk, V., Factor powers of finite symmetric groups, Math. Notes 58 (1995), 794-802.

8. Ganyushkin, O., and Mazorchuk, V., On the radical of $\mathscr{F}^{+}\left(S_{n}\right)$, Mat. Stud. 20 (2003), 17-26.

9. Ganyushkin, O., and Mazorchuk, V., Classical finite transformation semigroups. An introduction, Algebra Appl. 9, Springer, Berlin 2009.

10. Grood, C., The rook partition algebra, J. Combin. Theory Ser. A 113 (2006), 325-351.

11. Halverson, T., and Lewandowski, T., RSK insertion for set partitions and diagram algebras, Electron. J. Combin. 11 (2004/06), Research Paper 24, 24 pp.

12. Kim, K., and Roush, F., Two-generator semigroups of binary relations, J. Math. Psych. 17 (1978), 236-246.

13. Koenig, S., A panorama of diagram algebras, pp. 491-540 in: Trends in representation theory of algebras and related topics, EMS Ser. Congr. Rep., Eur. Math. Soc., Zürich 2008. 
14. Konieczny, J., Green's equivalences in finite semigroups of binary relations, Semigroup Forum 48 (1994), 235-252.

15. Kudryavtseva, G., and Mazorchuk, V., Partialization of categories and inverse braid-permutation monoids, Internat. J. Algebra Comput. 18 (2008), 989-1017.

16. Martin, P., Temperley-Lieb algebras for nonplanar statistical mechanics-the partition algebra construction, J. Knot Theory Ramifications 3 (1994), 51-82.

17. Martin, P., Potts models and related problems in statistical mechanics, Ser. Adv. Statistical Mech. 5, World Sci., Teaneck 1991.

18. Martin, P., On diagram categories, representation theory and Statistical Mechanics, pp. 99136 in: Noncommutative rings, group rings, diagram algebras and their rep., Contemporary Math 456, Amer. Math. Soc., Providence 2008.

19. Martin, P., and Mazorchuk, V., On the representation theory of partial Brauer algebras, to appear.

20. Mazorchuk, V., On the structure of the Brauer semigroup and its partial analogue, Problems in Algebra (Gomel) 13 (1998), 29-45.

21. Mazorchuk, V., Endomorphisms of $\mathfrak{B}_{n}, \mathfrak{B}_{n}$, and $\mathfrak{E}_{n}$, Comm. Algebra 30 (2002), 3489-3513.

22. Montague, J., and Plemmons, R., Maximal subgroups of the semigroup of relations J. Algebra 13 (1969), 575-587.

23. Plemmons, R., and West, M., On the semigroup of binary relations, Pacific J. Math. 35 (1970), 743-753.

24. Reshetikhin, N., and Turaev, V., Ribbon graphs and their invariants derived from quantum groups, Comm. Math. Phys. 127 (1990), 1-26.

25. Schwarz, S., On the semigroup of binary relations on a finite set, Czechoslovak Math. J. 20 (1970), 632-679.

26. Temperley, H., and Lieb, E., Relations between the "percolation” and “colouring” problem and other graph-theoretical problems associated with regular planar lattices: some exact results for the “percolation” problem, Proc. Roy. Soc. London Ser. A 322 (1971), 251-280.

27. Turaev, V., The category of oriented tangles and its representations, Funct. Anal. Appl. 23 (1989), 254-255.

28. Turaev, V., Quantum invariants of knots and 3-manifolds, 2 rev. ed., Gruyter Stud. Math. 18, de Gruyter, Berlin 2010.

DEPARTMENT OF PURE MATHEMATICS

UNIVERSITY OF LEEDS

LEEDS, LS2 9JT

UNITED KINGDOM

E-mail: pmartin@maths.leeds.ac.uk
DEPARTMENT OF MATHEMATICS UPPSALA UNIVERSITY

BOX. 480

SE-75106 UPPSALA

SWEDEN

E-mail: mazor@math.uu.se 